\title{
A novel interventional guidance framework for transseptal puncture in left atrial interventions
}

\author{
Pedro Morais ${ }^{1,2,3,4}$, João L. Vilaçaa ${ }^{3,4,5}$, Sandro Queirós ${ }^{2,3,4,6}$, Pedro L. \\ Rodrigues $^{3,4}$, João Manuel R. S. Tavares ${ }^{1}$, Jan D'hooge ${ }^{2}$ \\ ${ }^{1}$ Instituto de Ciência e Inovação em Engenharia Mecânica e Engenharia Industrial, \\ Departamento de Engenharia Mecânica, Faculdade de Engenharia, Universidade do Porto, \\ Porto, Portugal; \\ ${ }^{2}$ Lab on Cardiovascular Imaging \& Dynamics, Department of Cardiovascular Sciences, \\ KULeuven - University of Leuven, Leuven, Belgium; \\ ${ }^{3}$ Life and Health Sciences Research Institute (ICVS), School of Medicine, University of \\ Minho, Braga, Portugal; \\ ${ }^{4}$ ICVS/3B's-PT, Government Associate Laboratory, Braga/Guimarães, Portugal; \\ ${ }^{5}$ Ai-Technology School, Polytechnic Institute of Cávado and Ave, Barcelos, Portugal. \\ ${ }^{6}$ Algoritmi Center, School of Engineering, University of Minho, Guimarães, Portugal;
}

\begin{abstract}
Access to the left atrium is required for several percutaneous cardiac interventions. In these procedures, the inter-atrial septal wall is punctured using a catheter inserted in the right atrium via the venous system under image guidance. Although this approach (termed transseptal puncture - TSP) is performed daily, complications are common. Moreover, the exact location at which the septum needs to be traversed is determined entirely based on the interventionist's experience, which is sub-optimal. In this work, we present a novel concept for the development of an interventional guidance framework for TSP. The pre-procedural planning stage is fused with $3 D$ intra-procedural images (echocardiography) using manually defined landmarks, transferring the relevant anatomical landmarks to the interventional space and enhancing the echocardiographic images. In addition, electromagnetic sensors are attached to the surgical instruments, tracking them and allowing the inclusion of their spatial position in the enhanced intra-procedural world. Two patient-specific atrial phantom models were used to evaluate this framework. One operator performed the planning, calibrated the setup and performed the puncture. To assess the framework's accuracy, a metallic landmark was positioned in the punctured location and compared with the ideal one. The intervention was possible in both models, but in one case positioning of the landmark failed. An error of approximately of $6 \mathrm{~mm}$ was registered for the successful case. Technical characteristics of the framework showed an acceptable performance, with a frame rate $\sim 5$ frames/sec. The manual calibration setup required $\sim 60 \mathrm{~min}$. This study presented a proof-of-concept for an interventional guidance framework for TSP. However, a more automated solution and further studies to assess its accuracy are required.
\end{abstract}

Keyword Image-guided interventions; kidney; percutaneous renal access; porcine phantom. 


\section{Introduction}

Access to the left atrium (LA) is mandatory in multiple minimally invasive cardiac interventions, such as left atrial appendage closure, atrial fibrillation ablation, mitral valve replacement, among others $[1,2]$. Since no direct percutaneous access route to LA is available, a transseptal via is typically used. For that, a medical technique termed transseptal puncture (TSP) is applied, where a catheter is inserted via the femoral vein until the right atrium (RA), through which a needle is moved forward to puncture the inter-atrial septal (IAS) wall (using its thinnest region, the fossa ovalis - FO) and consequently gain access to the LA body [2]. The entire procedure is guided using medical images, namely fluoroscopy and echocardiography (mainly transesophageal echocardiography - TEE) [1]. Nevertheless, the success of the intervention is still highly dependent on the operator's expertise, which is sub-optimal. Indeed, when puncturing the IAS, not only the FO needs to be identified, but also the target location at the left heart and the catheter dexterity at this region must be taken into consideration, hampering the identification of the optimal puncture location [1].

To improve the TSP intervention, different technological innovations were presented during the last years. Three major development fields can be considered, namely: surgical tools, pre-procedural planning techniques and guidance approaches [1]. A high number of researchers focused on the former, presenting novel radio-frequency/electrocautery needles (instead of the traditional mechanical ones), which proved their clear advantages for abnormal situations [2]. Regarding the planning techniques, a small number of studies were presented, focusing on biomechanical simulation of the intervention [3] or automated identification of relevant landmarks (e.g. fossa ovalis position) [4], making the planning stage faster and more reproducible. Regarding the intraoperative guidance, several researchers explored the potential use of novel imaging modalities (beyond the traditional ones, magnetic resonance imaging - MRI, and intracardiac echocardiography) for TSP $[1,6]$. Moreover, electroanatomical mapping solutions or even electromagnetic guidance solutions were also described [1]. More recently, some researchers presented imagefusion strategies [6-8], where the bidimensional and low contrast fluoroscopic image is fused with $3 \mathrm{D}$ anatomical detailed models (extracted from echocardiography or computed tomography - CT), showing clear advantages for TSP with inferior procedural time and higher success rate in difficult cases. Nevertheless, although such image fusion solutions showed high potential to ease the intervention [6-8], most of them fuse intra-procedural images only (not allowing the inclusion of preprocedural planning information) or were not validated for TSP.

In this study, we present a novel concept for the development of an integrated interventional planning and guidance framework to assist the physician in successfully performing TSP. 


\section{Methods}

The proposed interventional framework is divided into (Fig. 1): 1) the pre-procedural and 2) the intra-procedural stages. During the first stage, identification or delineation (step A) of relevant cardiac chambers in a highly-detailed image (CT) is performed. Then, based on the estimated contours, the full extent of the FO is estimated and the optimal puncture location is defined by the expert (step B). The entire planning information is then transferred to the intra-procedural world (step C), by fusing intra- and pre-procedural data (e.g. contours or landmarks). Note that intraprocedural data is extracted from echocardiographic images only (in this initial setup, transthoracic echocardiography - TTE - was used). Finally, to also include the surgical instruments into this augmented environment, a tracking strategy is applied using external electromagnetic sensors (step D). An initial calibration between the TTE image world and the electromagnetic sensors was required (step E). By combining all these elements (step F), a radiation-free interventional framework with enhanced anatomical information (from the planning stage) is achieved.

\section{Interventional Framework}

The interventional framework was implemented in $\mathrm{C}++$ and it exploits the potentialities of the VTK (Visualization Toolkit) library [11] for the visualization of images/surfaces and even 3D rendering (using OpenGL). The framework has 4 independent views (see Figure 2), allowing the visualization of the pre- and intraprocedural data through $2 \mathrm{D}$ views or $3 \mathrm{D}$ renderings. The current version implements the intra-procedural guidance stage only, presenting import functions to include the pre-procedural planning data. Moreover, specific libraries to receive, in real-time, 3D TTE images (from a commercially available ultrasound - US machine) and the 3D position of the different instruments were used.

As such, the different steps of the described concept were implemented as (Fig. 2):

Step A: A manual delineation of the LA and RA was performed using the Medical Imaging Interaction Toolkit (MITK) software. In detail, multiple 2D slices were delineated and then interpolated into a 3D surface. Each surface was independently delineated and saved in stl (stereolitrography) format.

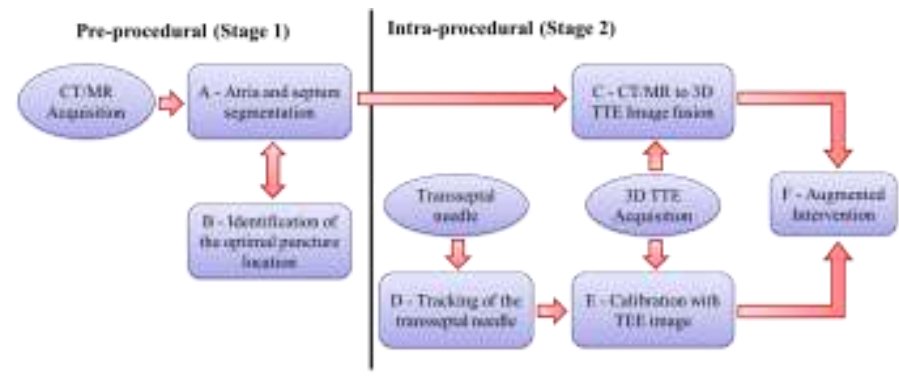

Figure 1 - Blocks diagram of the proposed concept. 


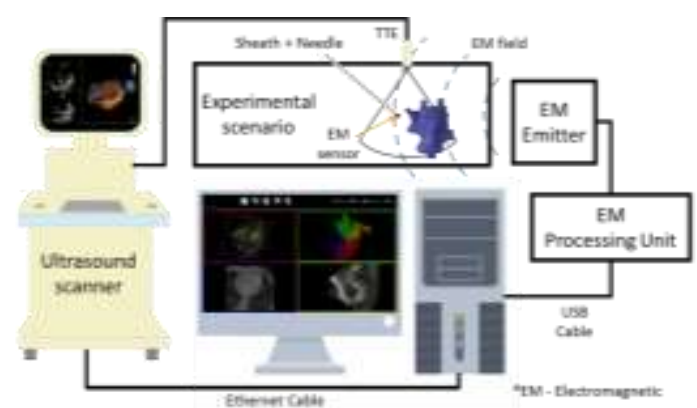

Figure 2 - Overview of the developed interventional setup.

Step B: Based on the 3D contours from (A), the FO was manually identified. For that, we detected the thinnest region, as described in [4]. Then, the optimal puncture location was marked and saved in stl format.

Step C: Both pre-procedural (CT) and intra-procedural (TTE) images were uploaded and streamed in the described framework (Fig. 2), respectively. The CT image is uploaded using the DICOM (Digital Imaging and Communications in Medicine) read function currently available in VTK. In opposition, the TTE images were acquired in real-time with a Vivid E95 (GE Vingmed, Horten, Norway) scanner, equipped with a $4 \mathrm{~V}$-D transducer and streamed using a proprietary software. Regarding the image-fusion between CT and TTE worlds, the following strategy was applied. By visualizing both images in parallel, a set of landmarks were manually defined in both images, being later used to fuse both image coordinate space. The optimal transformation between CT-TTE worlds was computed through a leastsquare strategy. After estimating the optimal transformation, the surfaces generated throughout steps A and B are imported and automatically superimposed on the intraprocedural image, enhancing the relevant anatomical landmarks.

Step D: A small electromagnetic sensor (EM, Fig. 2) with 6 degrees of freedom (DOF), Aurora 6DOF Flex Tube, Type 2 (Aurora, Northern Digital, Waterloo, Ontario), was attached to the tip of the transseptal sheath.

Step E - A fixed calibration was made to combine the electromagnetic and TTE worlds. In this sense, a set of positions were identified in the TTE image. Then, the same spatial positions were physically achieved by the EM sensor, and the final optimal transformation was obtained by applying a least-square fitting between all positions. By applying this spatial transformation, a unique scenario combining the enhanced intra-procedural image with the needle position was obtained, allowing the correct guidance of the surgical tool until the optimal puncture location. Inside the proposed guidance framework, the needle position was represented as a red dot.

\section{Experiments}

Description: In this version, two patient-specific mock models of the atria were used (Fig. 3). Both static models were constructed using the strategy described in [10]. 
Implementation Details: Since mock models were used, the TTE probe was kept fixed. Before executing the calibration steps, one operator selected the optimal field of the view (FOV) of the model. Regarding the identification of relevant landmarks (required in step C and E), Fig. 4 presents an overview of the target positions.

Evaluation: One operator applied the described pipeline in each phantom model and then performed a TSP. To evaluate the error between the selected location and the puncture position, a metallic landmark was later inserted to mark the punctured site. Later, a CT acquisition of the model plus the landmark was acquired. This postinterventional CT was segmented and the obtained surfaces were aligned with the planning surfaces using an iterative closest point algorithm. Finally, the frame rate achieved by the framework for the streaming of US data was also evaluated. All results were computed using a personal laptop with Intel (R) i7 CPU at $2.8 \mathrm{GHz}$ and 16 GB of RAM. An integrated graphics card Nvidia Quadro K2100 was used.

\section{Results}

The TSP was possible in both cases. Overall, guidance with this setup was considered challenging. In one phantom model, an error of approximately $6 \mathrm{~mm}$ was found between the selected position and the metallic landmark. For the second model, it was not possible to insert the metallic landmark. Regarding the technical characteristics, a frame rate of approximately 5 frames/second was achieved. The technical calibration took $\sim 60 \mathrm{~min}$. The planning stage required $\sim 30 \mathrm{~min}$.

\section{Discussion}

In this study, a novel interventional framework for TSP is described. It uses the potentialities of the intra-procedural volumetric US image to create an integrated interventional scenario where both pre-planning, intra-procedural data, and surgical

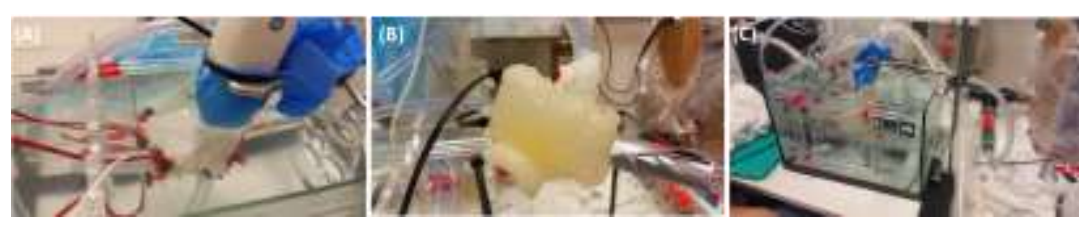

Figure 3 - Experimental validation scenario.

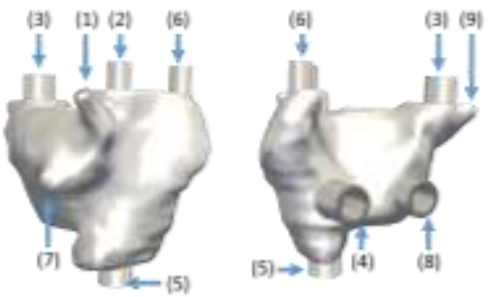

Figure 4 - Relevant landmark positions for step C and E. 
instrument position are fused. Thus, the not well contrasted and noisy TTE image is enhanced by superimposing virtual anatomical surfaces. Moreover, the optimal puncture location can also be visualized, potentially increasing the safety of the intervention. In opposition to other studies [11], the current concept allows the inclusion of pre-procedural planning information in the interventional world. Indeed, recent solutions, such as the EchoNavigator (Philips Inc., Netherlands), [12] proved its added-value for TSP intervention, by adding anatomical information (US images) to the fluoroscopy. Nevertheless, although this solution allows the inclusion of specific landmarks in the interventional image, one is not able to embed preprocedural planning information in the intra-procedural world [12]. In a different way, CT-fluoroscopy fusion approaches were also described and validated for TSP [7], therefore the usage of pre-procedural data into the interventional scenario. However, since the US image is not integrated, relevant online anatomical details are lost or ultimately require an independent US scanner during the intervention.

This framework has as a key novelty the direct usage of 3D US data (by streaming it) to fuse intra-procedural data with pre-procedural one. In fact, previous works focused on similar methodologies for different scenarios. Nevertheless, 2D US data was mainly streamed, requiring complex calibration scenarios to perform 2D-3D alignment $[11,14]$. Although such approaches have shown interesting results in nearly static structures/organs [12], its application in cardiac interventions is limited. As such, by capturing the entire 3D volume, 2D-3D alignment/reconstruction steps are removed, potentially improving the performance and accuracy of imagefusion algorithms. However, the described pipeline is only an initial proof-of-concept and it still presents some drawbacks, namely manual interaction is mandatory in all stages, making the configuration of the setup extremely time-consuming. Recently, our team has presented different methodologies to automate the this framework: 1) automatic segmentation of the atrial region in CT [4, 15]; 2) automatic identification of the FO in CT [4]; and 3) automatic segmentation of the LA [16]. As such, the entire planning can be performed quickly (2-3 min, [4]) and the fusion stage can be quickly performed by aligning the segmented models. Although the current automated modules are not integrated into this framework, such options are expected in a future release. Regarding the tracking of the different surgical tools, fixed calibration setups can be used [11], making its calibration fast. Nevertheless, to improve the guidance of the surgical tools and the identification of the optimal puncture route, two modifications should be performed to the current setup: i) multiple sensors should be embedded along the instrument, providing a virtual representation of the entire instrument's shape inside the body, and ii) an enhanced representation of the optimal puncture route can potentiate the guidance stage and facilitate the identification of the puncture location. Finally, regarding the frame rate, an acceptable performance ( 5 frames/second) was achieved by this framework. The obtained results showed that accurate evaluation of the proposed framework was not possible. First, a small number of phantom models were used. Second, the strategy applied to mark the punctured location proved to be sub-optimal. Due to 
the small entry points of the phantom model, visual identification punctured location was challenging, hampering the insertion of the metallic landmark. Third, quantification of the framework's accuracy through the described approach (i.e. aligning post-interventional data with pre-interventional one) is sensitive to small alignment errors. In this sense and to improve the described experiment, a novel scenario is required with the following features: 1) a large number of phantom models with different anatomies are required; 2 ) inclusion of radiopaque materials to easily allow an accurate alignment between the pre- and pos- interventional surfaces could be a potential solution; and 3) instead of using metallic landmarks, the TSP needle should be kept at the punctured location. Finally, since the traditional intervention is widely dependent of the fluoroscopy, further studies to evaluate the feasibility of this potential radiation-free framework and even to evaluate the required learning curve are mandatory to validate it. Regarding the study limitations, we would like to emphasize that: 1) static phantom models were used;2) instead of a TEE probe, a TTE one was used; and 3) the US probe was kept fixed throughout the intervention. To overcome these limitations, dynamic phantom setups, as described in [10], should be used, and an electromagnetic sensor should be attached to the ultrasound probe (as described in [14]), spatially relating the ultrasound FOV with the probe position and allowing its free manipulation throughout the intervention.

\section{Conclusion}

The described concept for the development of an interventional guidance framework showed its initial potential usefulness for the identification of the optimal puncture location and to guide the TSP intervention. Nevertheless, the current version requires manual interaction in all stages, making the configuration setup extremely time-consuming and difficult to be performed. Further studies and a different experimental setup are required to accurately validate the proposed framework.

\section{Acknowledgments}

The authors acknowledge Fundação para a Ciência e a Tecnologia (FCT), in Portugal, and the European Social Found, European Union, for funding support through the "Programa Operacional Capital Humano" (POCH) in the scope of the PhD grants SFRH/BD/95438/2013 (P. Morais) and SFRH/BD/93443/2013 (S. Queirós). This work was funded by projects NORTE-01-0145-FEDER-000013, NORTE-010145-FEDER-000022 and NORTE-01-0145-FEDER-024300, supported by Northern Portugal Regional Operational Programme (Norte2020), under the Portugal 2020 Partnership Agreement, through the European Regional Development Fund (FEDER), and has also been funded by FEDER funds, through Competitiveness Factors Operational Programme (COMPETE), and by national funds, through the FCT, under the scope of the project POCI-01-0145-FEDER-007038.

The authors would like to acknowledge Walter Coudyzer and Steven Dymarkowski 
(Department of Radiology, UZLeuven, Leuven, Belgium) for performing the CT acquisitions. Moreover, the authors would like to thank General Electric (GE VingMed, Horten, Norway) for giving access to the 3D streaming option.

\section{References}

[1] P. Morais, J. L. Vilaça, J. Ector, J. D'hooge, and J. M. R. S. Tavares, "Novel solutions applied in transseptal puncture: a systematic review," Journal of Medical Devices, vol. 11, p. 010801, 2017.

[2] J. C. Hsu, N. Badhwar, E. P. Gerstenfeld, R. J. Lee, et al., "Randomized Trial of Conventional Transseptal Needle Versus Radiofrequency Energy Needle Puncture for Left Atrial Access" Journal of the American Heart Association, vol. 2, p. e000428, 2013.

[3] J. Jayender, R. V. Patel, G. F. Michaud, and N. Hata, "Optimal transseptal puncture location for robot-assisted left atrial catheter ablation," The International Journal of Medical Robotics and Computer Assisted Surgery, vol. 7, pp. 193-201, 2011.

[4] P. Morais, J. L. Vilaça, S. Queirós, A. Marchi, F. Bourier, et al., "Automated segmentation of the atrial region and fossa ovalis towards computer-aided planning of inter-atrial wall interventions," Computer Methods and Programs in Biomedicine, 2018.

[5] C. P. Ruisi, N. Brysiewicz, J. D. Asnes, L. Sugeng, M. Marieb, J. Clancy, et al., "Use of intracardiac echocardiography during atrial fibrillation ablation," Pacing and Clinical Electrophysiology, vol. 36, pp. 781-788, 2013.

[6] P. Biaggi, C. Fernandez-Golfín, R. Hahn, and R. Corti, "Hybrid imaging during transcatheter structural heart interventions," Current cardiovascular imaging reports, vol. 8, p. 33, 2015.

[7] F. Bourier, T. Reents, S. Ammar-Busch, V. Semmler, M. Telishevska, M. Kottmaier, et al., "Transseptal puncture guided by CT-derived 3D-augmented fluoroscopy," Journal of cardiovascular electrophysiology, vol. 27, pp. 369-372, 2016.

[8] S. Afzal, V. Veulemans, J. Balzer, T. Rassaf, K. Hellhammer, A. Polzin, et al., "Safety and efficacy of transseptal puncture guided by real-time fusion of echocardiography and fluoroscopy," Netherlands Heart Journal, vol. 25, pp. 131-136, 2017.

[9] W. J. Schroeder, B. Lorensen, and K. Martin, The visualization toolkit: an object-oriented approach to 3D graphics: Kitware, 2004.

[10] P. Morais, J. M. R. Tavares, S. Queirós, F. Veloso, J. D'hooge, and J. L. Vilaça, "Development of a patient-specific atrial phantom model for planning and training of inter-atrial interventions," Medical physics, vol. 44, pp. 5638-5649, 2017.

[11] K. Cleary and T. M. Peters, "Image-guided interventions: technology review and clinical applications," Annual review of biomedical engineering, vol. 12, pp. 119-142, 2010.

[12] F. F. Faletra, L. Biasco, G. Pedrazzini, M. Moccetti, et al., "Echocardiographic-Fluoroscopic Fusion Imaging in Transseptal Puncture: A New Technology for an Old Procedure," Journal of the American Society of Echocardiography, vol. 30, pp. 886-895, 2017.

[13] R. J. Housden, M. Basra, Y. Ma, A. P. King, R. Bullens, N. Child, et al., "Three-modality registration for guidance of minimally invasive cardiac interventions," in Functional Imaging and Modeling of the Heart, ed: Springer, 2013, pp. 158-165.

[14] P. Lang, P. Seslija, M. W. Chu, D. Bainbridge, G. M. Guiraudon, D. L. Jones, et al., "USfluoroscopy registration for transcatheter aortic valve implantation," IEEE Transactions on Biomedical Engineering, vol. 59, pp. 1444-1453, 2012.

[15] P. Morais, J. L. Vilaça, S. Queirós, F. Bourier, I. Deisenhofer, J. M. R. S. Tavares, et al., "A competitive strategy for atrial and aortic tract segmentation based on deformable models," Medical Image Analysis, vol. 42, pp. 102-116, 12// 2017.

[16] N. Almeida, D. Friboulet, S. I. Sarvari, O. Bernard., "Left-Atrial Segmentation From 3D Ultrasound Using B-Spline Explicit Active Surfaces With Scale Uncoupling," IEEE transactions on ultrasonics, ferroelectrics, and frequency control, vol. 63, 2016. 